\title{
焦磷酸酯合成的研究进展
}

\author{
侯淑华* 曲忠国汤立军 \\ (渤海大学化学化工学院 辽宁省功能化合物的合成与应用重点实验室 锦州 121013)
}

\begin{abstract}
摘要 综述了焦磷酸酯类化合物的合成方法研究进展，分别按照不同的活化方法进行了阐述，包括二环己基碳二亚胺 (DCC), $N, N$-羰基二咪唑(CDI), DCC/吗啉 $/ \mathrm{MnCl}_{2} / \mathrm{MgSO}_{4}, \mathrm{DCC} /$ 吗啉 $/ 1 H$-四唑, $\mathrm{PPh}_{3} /(\mathrm{PyS})_{2} / N$-甲基咪唑，磷酰氯二苯酯 (DPCP), 三氟乙酸酐(TFAA) $/ N$-甲基咪唑，并对部分反应机理进行了讨论.

关键词 焦磷酸酯; 合成; 进展
\end{abstract}

\section{Advances in the Synthesis of Organic Pyrophosphate}

\author{
Hou, Shuhua* Qu, Zhongguo Tang, Lijun \\ (Department of Chemistry, Liaoning Key Laboratory for the Synthesis and Application of Functional Compounds, \\ Bohai University, Jinzhou 121013)
}

\begin{abstract}
The recent advances in the synthesis of organic pyrophosphate are reviewed, based on the coupling methods including dicyclohexylcarbodiimide (DCC), $N, N^{\prime}$-carbonyldiimidazole (CDI), DCC/morpholine/ $\mathrm{MnCl}_{2} / \mathrm{MgSO}_{4}, \mathrm{DCC} /$ morpholine/ $1 H$-tetrazole, $\mathrm{PPh}_{3} /(\mathrm{PyS})_{2} / N$-methylimidazole, diphenyl phosphorochloridate (DPCP), trifluoroacetic anhydride/ $N$-methylimidazole. Mechanism of some reactions is also discussed.
\end{abstract}

Keywords organic pyrophosphate; synthesis; advances

焦磷酸酯普遍存在于生物界中, 是生命活动的重要 参与者. 如烟酰胺腺嘌呤二核苷酸(nicotinamide adenine dinucleotide, $\mathrm{NAD}^{+}$, Scheme 1), 它在生物体中起到传递 氢和电子的作用, 以保持细胞内氧化还原平衡, 同时参 与能量传递, 物质代谢和信号传导等过程 ${ }^{[1]}$. 糖焦磷酸 核苷酯(Sugar-nucleotide, Scheme 1)是碳水化合物代谢、 蛋白质和油脂等天然分子糖基化的重要中间体 ${ }^{[2]}$, 如 UDP- $\beta$ - $L$-rhamnose (Scheme 2 ) 是植物细胞中胶质及细胞 壁的合成的重要参与者 ${ }^{[3]}$; UDP- $D$-galactose 等 8 种糖焦 磷酸核苷酯(Scheme 2)可以合成真核生物中几乎所有糖 蛋白和糖脂 ${ }^{[4]}$. 这些分子具有一个共性, 都含有焦磷酸 键. 要探索这些分子在生物体内的作用, 常通过设计合 成其类似物来研究其性质. 目前, 焦磷酸酯的化学合成 主要存在以下困难: 对称型焦磷酸酯副产物的生成、在 有机溶剂中溶解度极低、高极性和带电荷基团存在于分 子结构中导致活化困难、涉及保护脱保护过程导致合成 步骤长、焦磷酸键及糖苷键容易水解断裂.

近十年有较多关于焦磷酸酯合成的文献报道, 也有 文章对糖焦磷酸核苷酯进行综述, 主要包括酶法 ${ }^{[5]}$ 和化

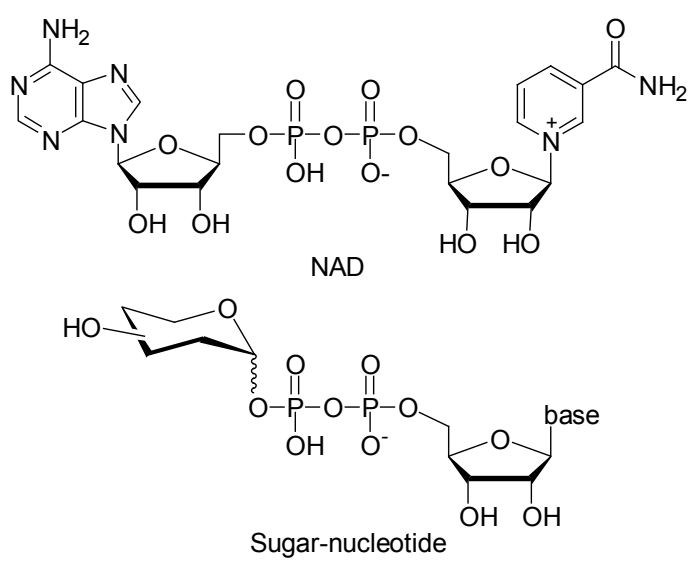

Scheme 1

学合成法 ${ }^{[6]}$, 设计合成类似物的片段可能并不被生物体 中合成焦磷酸酯的酶所识别, 因此酶法不一定适合所有 的焦磷酸酯的合成, 本文主要对合成焦磷酸酯的化学方 法进行概括. 合成焦磷酸酯可以选择不同的合成路线, 其逆合成分析如 Scheme 3.

*E-mail: shyspa0812@163.com

Received July 3, 2013; revised August 11, 2013; published online September 25, 2013. 


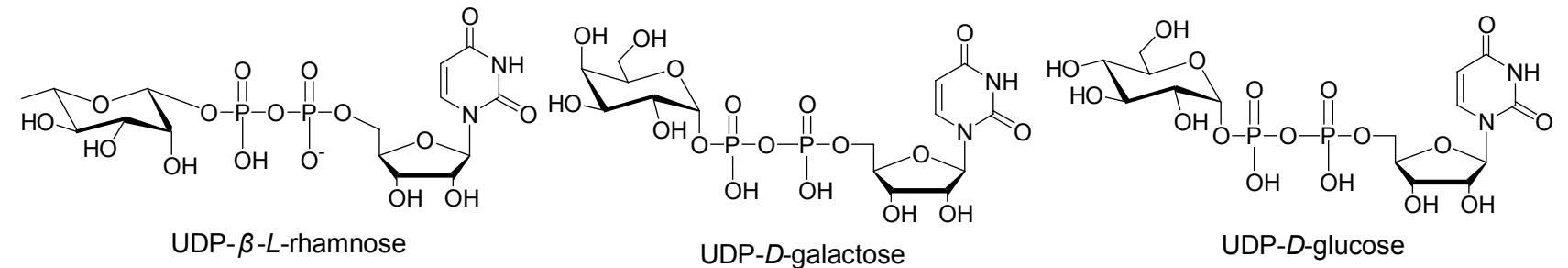

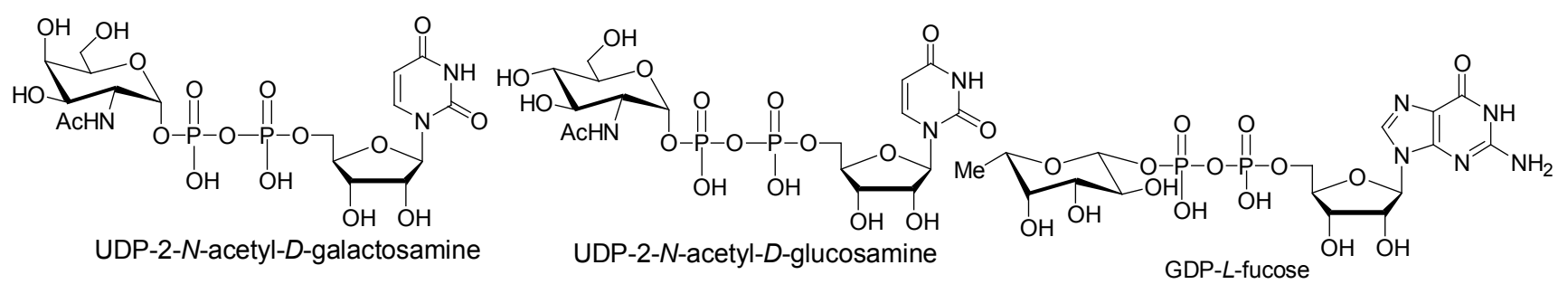

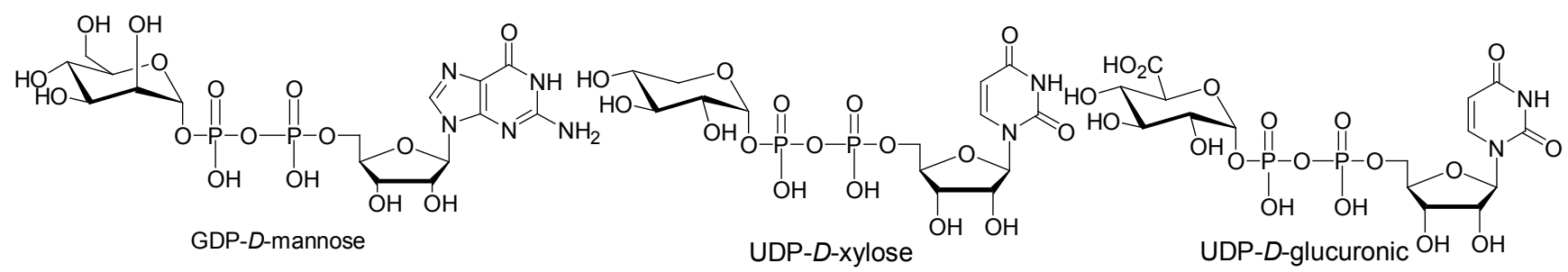

Scheme 2

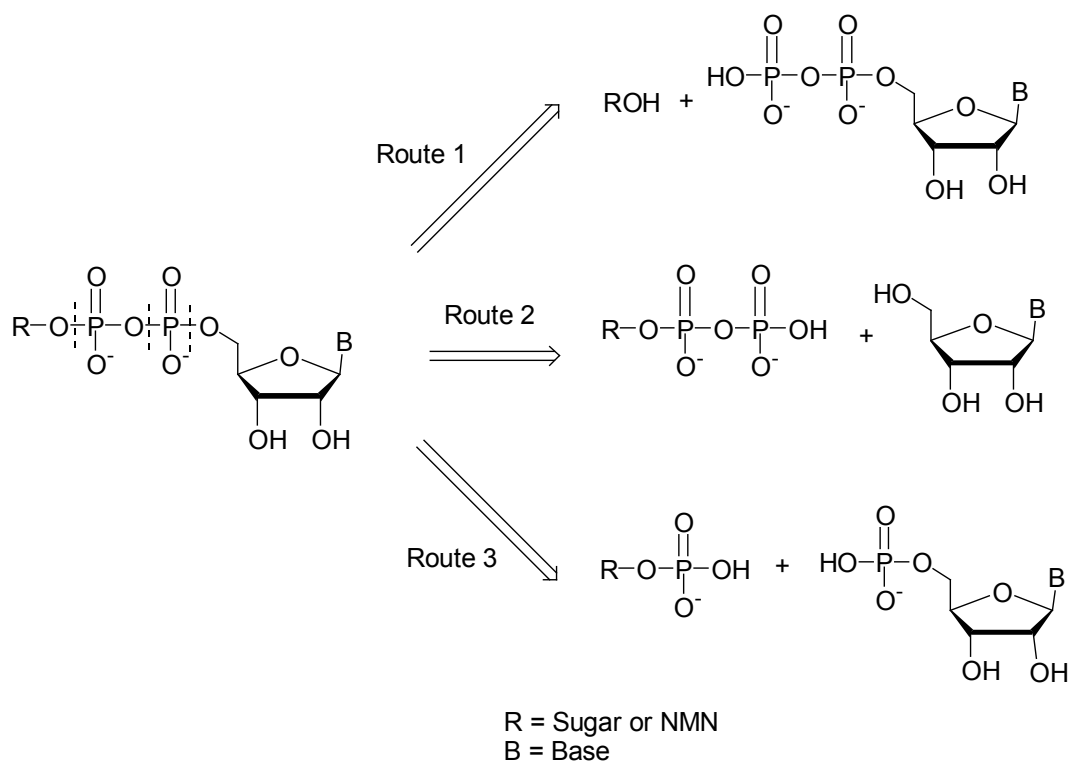

Scheme 3

对 3 种反合成路线分析, Route 1 和 Route 2 需要先 合成焦磷酸键, 焦磷酸键的合成需要活化试剂的参与, 而下一步焦磷酸与羟基的偶联同样需要活化试剂的参 与, 增加反应难度; Route 3 通过两种磷酸单酯合成, 只 需要一步活化, 降低合成难度, 且文献报道合成磷酸单 酯的方法比较多, 最具有可行性. Route 3 首先需要活化 一分子的磷酸单酯, 然后与另外一分子磷酸单酯反应 (Scheme 4). 活化反应的反应温度不能太高, 否则易造 成糖苷键断裂; 活化过程中要避免对称的焦磷酸酯生
成. 目前常用的活化磷酸单酯的试剂有: 二环己基碳二 亚胺 ( $N, N^{\prime}$-dicyclohexylcarbodiimide, DCC), $N, N$-羊炭基 二咪唑 $\left(N, N^{\prime}\right.$-carbonyldiimidazole, $\left.\mathrm{CDI}\right), \mathrm{DCC} /$ 吗啉/ $\mathrm{MnCl}_{2} / \mathrm{MgSO}_{4}, \mathrm{DCC} /$ 吗啉 $/ 1 \mathrm{H}$-四唑, 三苯基膦 (triphenylphosphine, $\mathrm{PPh}_{3}$ )/2,2-二硫二吡啶 $(\mathrm{PyS})_{2} / N$-甲 基咪唑, 磷酰氯二苯酯(diphenyl phosphorochloridate, DPCP), 三氟乙酸䣶 (Trifluoroacetic anhydride, $\mathrm{TFAA}) / N$-甲基咪唑. 


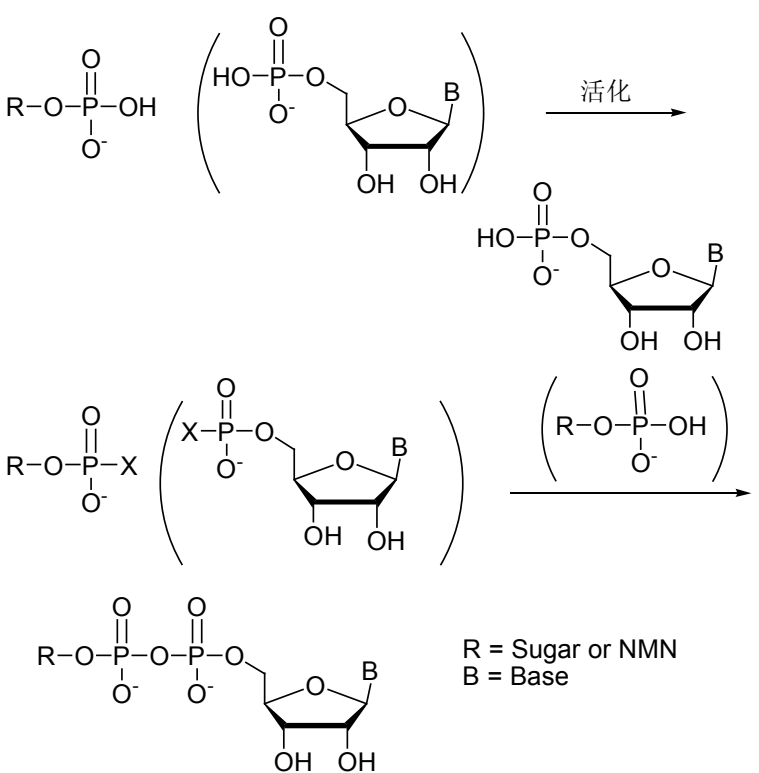

Scheme 4

\section{DCC}

Todd 等 ${ }^{[7]}$ 于 1957 年首次合成了 $\mathrm{NAD}$, 在 $75 \%$ 吡啶 水溶液中用 DCC 将烟酰胺单核苷酸(NMN) 和腺嘌呤单 核苷酸(AMP)偶联(Scheme 5). NMN 分子中含有一个正 电荷, 分子内形成内盐, 反应活性较低可以采用 DCC 获 得产物, 但是磷酸单酯与 DCC 形成的中间体在反应条 件下，很容易水解回到原料，再与中间体反应，生成对 称焦磷酸副产物, 且如果含有 $\mathrm{NH}_{2}, \mathrm{OH}, \mathrm{CO}_{2} \mathrm{H}$ 等活性基 团易发生副反应, 在焦磷酸酯的合成中需要复杂的保护 与脱保护过程.

\section{CDI}

$N, N$-羊炭基二咪唑是咪唑的衍生物, 具有较强的化学 反应活性，能与酸、氨、羟基等官能团反应，合成活泼 的活性中间体. 如活化磷酸单酯, 然后与另一分子磷酸

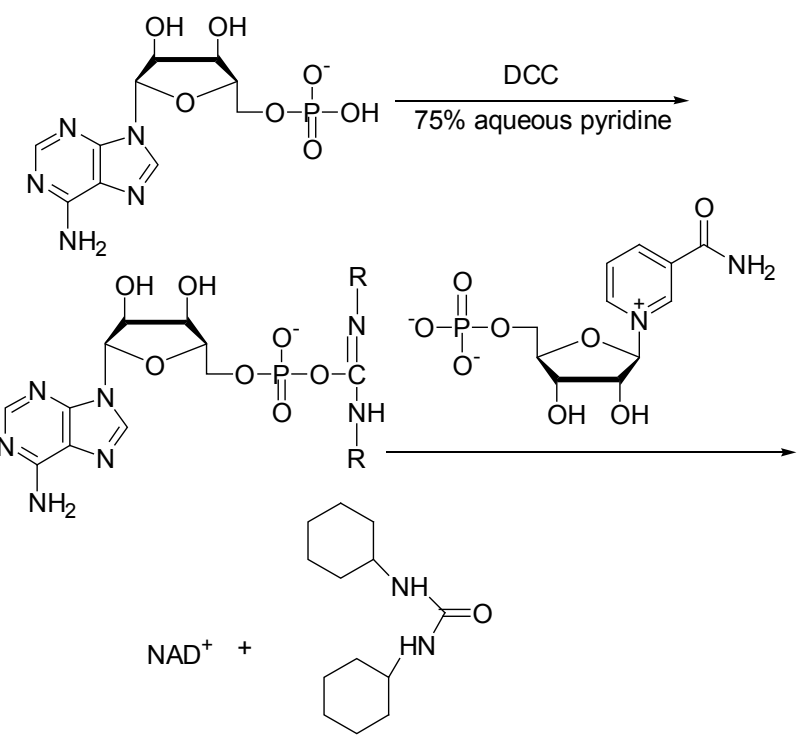

Scheme 5

单酯反应获得焦磷酸酯. 反应机理如 Scheme 6.

Dowden 等 ${ }^{[8]}$ 利用 CDI 活化 NMN 合成了 NADP (Scheme 7), 我们课题组 ${ }^{[9]}$ 利用 CDI 活化 NMN 合成 NAD 类似物(Scheme 8). CDI 具有较高的化学反应活性, 可以活化含有内盐的磷酸单酯，然后再进行下一步反 应.

$\mathrm{CDI}$ 除了用来合成焦磷酸型核酸及其类似物 ${ }^{[10]}$ ，也 用来合成糖焦磷酸核苷醌 ${ }^{[11]}$, Kurosu 等 ${ }^{[11 a]}$ 以 CDI 为活 化试剂活化糖磷酸单酯, 然后与核苷酸反应合成 UDP-MurNAc 来篎选膜转运蛋白(MraY)抑制剂(Scheme 9); Ko 等 ${ }^{[11 e]}$ 用 CDI 活化核苷酸然后再与未进行保护的 糖磷酸单酯反应制得 5'-焦磷酸尿嘧啶葡萄糖酯(Uridine 5'-diphosphoglucose)类似物(Eq. 1), 产率在 7\% 63\%之 间，这些类似物被用以作为人 $\mathrm{P} 2 \mathrm{Y}_{14}$ 抑制剂. 以 $\mathrm{CDI}$ 为 活化试剂，反应中间产物无需分离直接进行下一步反
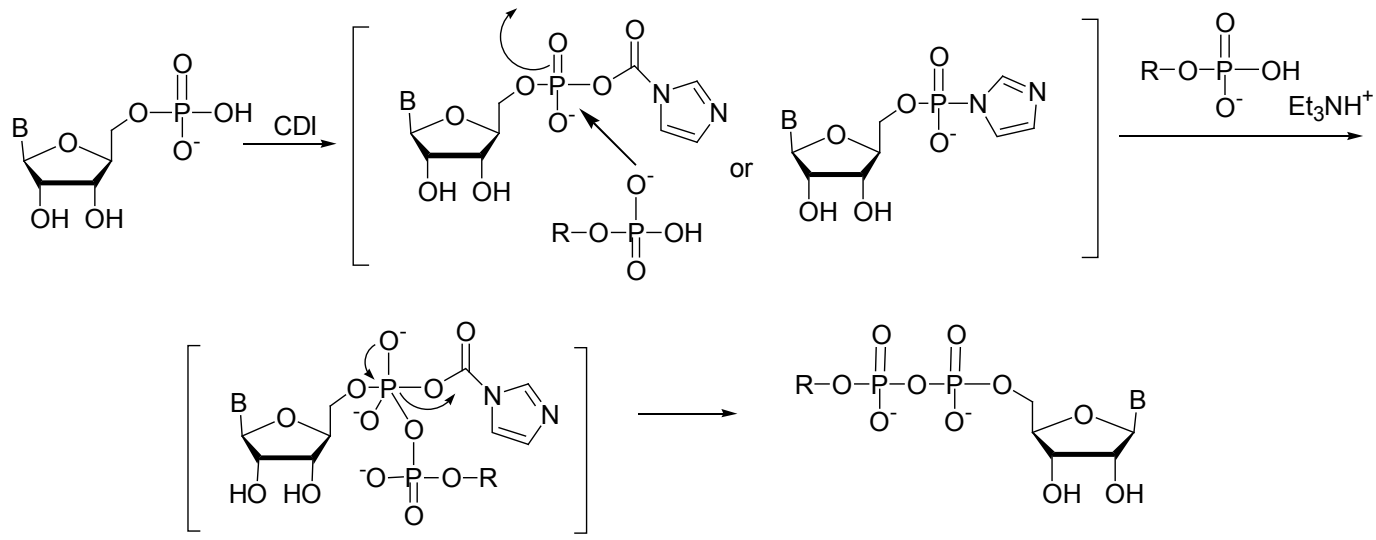

Scheme 6 


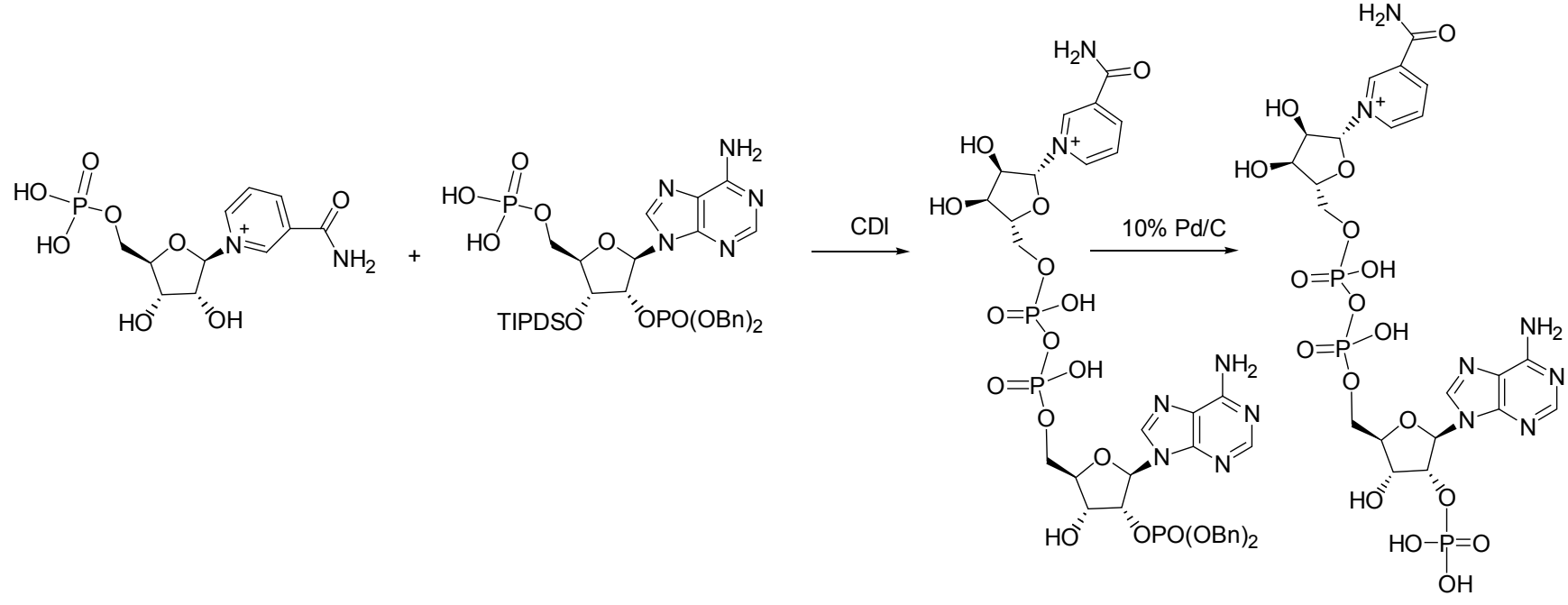

Scheme 7

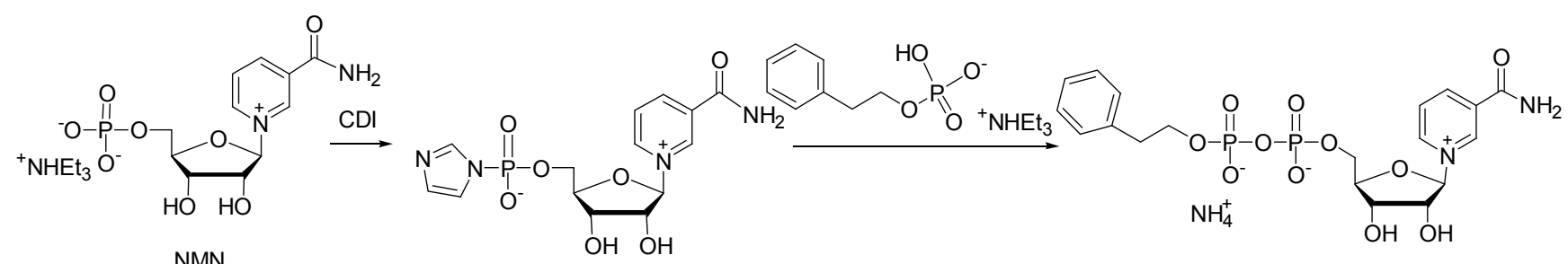

Scheme 8

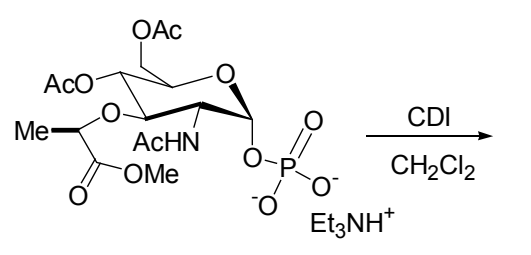

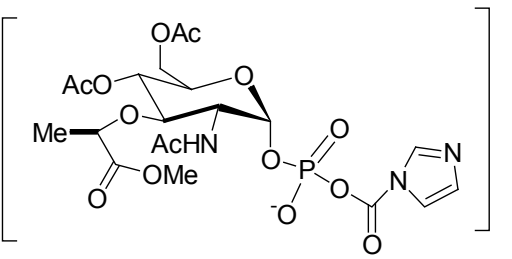

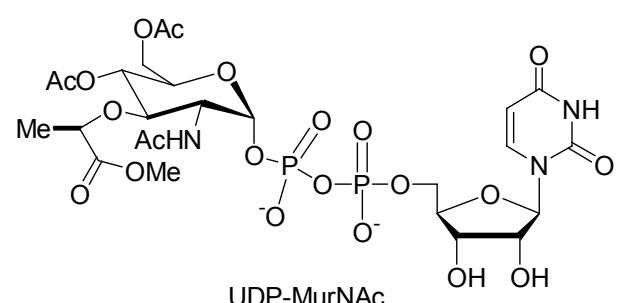<smiles>CCN(CC)OP(=O)([O-])OOC1OC2OC(=O)C1C2n1ccc(=O)[nH]c1=O</smiles>

\section{Scheme 9}

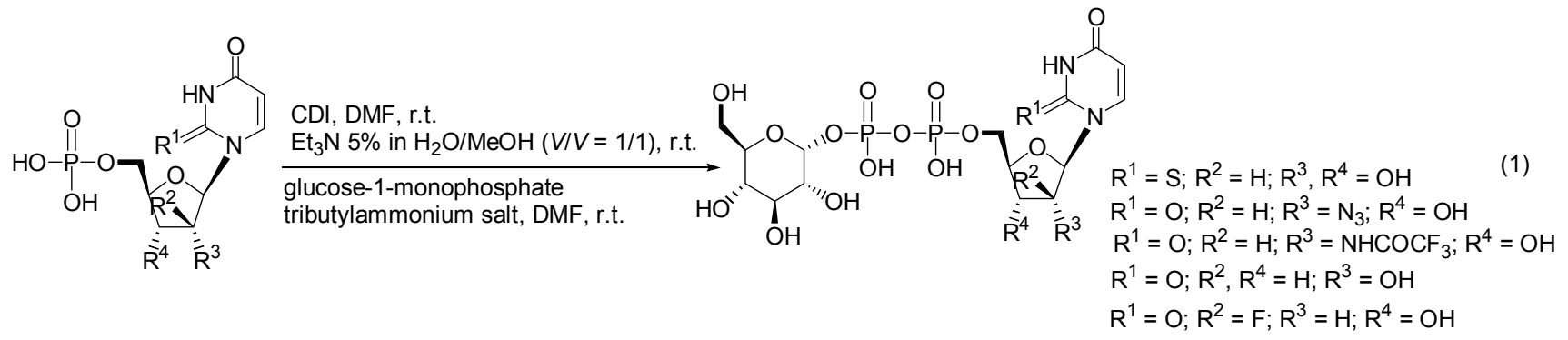


应，但是后续反应时间较长，一般需要 $2 \sim 3 \mathrm{~d}^{[10 b, 12]}$.

\section{$3 \mathrm{DCC} /$ 吗啉}

Khorana 等 ${ }^{[13]}$ 首先用活性中间体磷酰胺作为亲电试 剂, 与另外一分子磷酸单酯反应制备糖焦磷酸酯, 成功 制备了 UDP- $\alpha$ - $D$-glucose，高达 59\% 分离产率。后来 Khorana 课题组 ${ }^{[14]}$ 对磷酰胺中间体进行了改进, 发展了 具有更高反应活性的磷酰吗啉(phosphoromorpholidate) 中间体.

DCC 具有很高的反应活性, 只用 DCC 活化磷酸单 酯会形成对称的焦磷酸酯产物, 因此加入吗啉形成磷酰 吗啉中间体, 然后与另一分子磷酸单酯反应生成焦磷酸 酯，反应机理如 Scheme 10.<smiles>[R]OP(=O)(O)O</smiles><smiles>CC(=O)CC1CNCCO1</smiles>

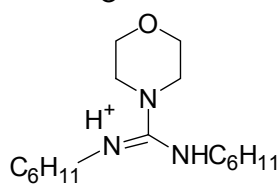<smiles>[R]OP(=O)([O-])[O-]</smiles>

催化剂

Scheme 10

磷酰吗啉中间体与另一分子的磷酸单酯反应时间 较长, 一般需要几天到几个星期, 为了缩短反应时间,
通常加入催化剂催化反应，常用的催化剂有 $\mathrm{MnCl}_{2}$ / $\mathrm{MgSO}_{4}$ 和 $1 H$-四唑，这两种催化剂被用来合成天然的及 非天然的活性分子.

\section{1 $\mathrm{MnCl}_{2} / \mathrm{MgSO}_{4}$}

糖焦磷酸核苷酯可以采用 $\mathrm{MnCl}_{2} / \mathrm{MgSO}_{4}$ 催化的方 法获得, Wagner 等 ${ }^{[15]}$ 采用路易斯酸催化的方法合成 8-phenyl GDP-Mannose (Eq. 2), 首先合成中间体磷酰吗 啉, 然后与一分子的甘露糖 1-磷酸反应，获得产物，产 率 $44 \%$. 催化反应的机理是锰离子与磷酰胺中的氮络 合，使离去基团吗啉易于离去.
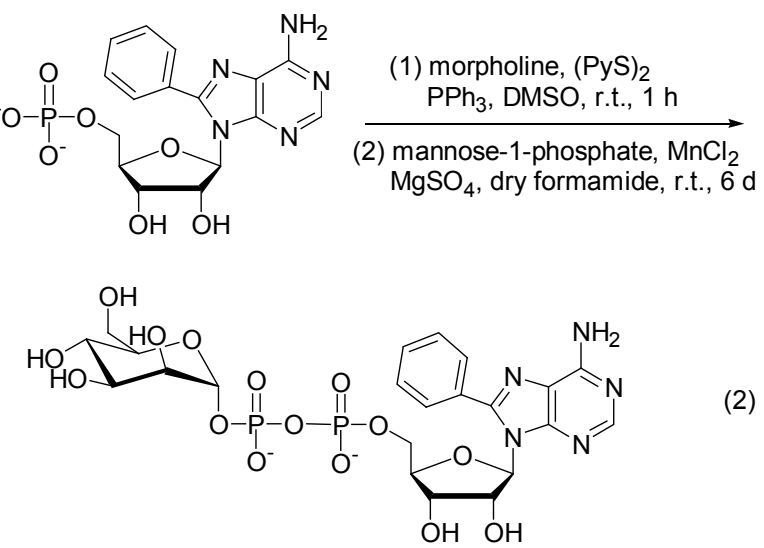

8-phenyl GDP-mannose

Lee 等 ${ }^{[16]}$ 首先采用路易斯酸催化合成 NAD (Scheme 11)，此后这种方法被普遍应用到 NAD 类似物上(表 1), 此种方法具有反应时间较短 $(1 \sim 2 \mathrm{~d})$ 、收率较高、操作 简单，价格便宜的优点.

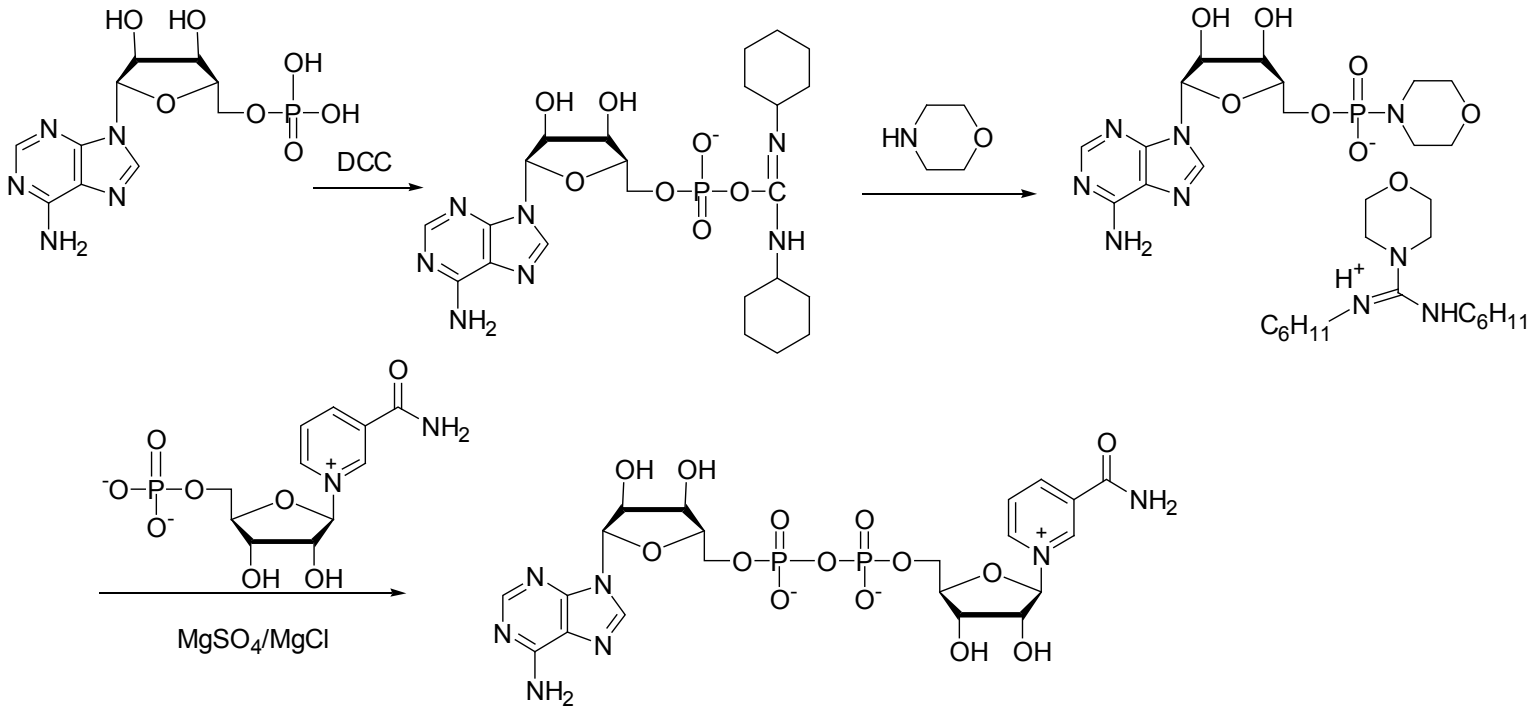

Scheme 11 
表1 路易斯酸 $\mathrm{MnCl}_{2} / \mathrm{MgSO}_{4}$ 催化合成NAD ${ }^{+}$类似物

Table 1 Lewis acid $\mathrm{MnCl}_{2} / \mathrm{MgSO}_{4}$ catalysis for $\mathrm{NAD}^{+}$analogs synthesis

\begin{tabular}{|c|c|c|c|}
\hline $\mathrm{NAD}^{+}$analog & Reaction time & Yield $/ \%$ & Reference \\
\hline $\begin{array}{l}\text { Disubstituted } \\
\mathrm{NAD}^{+} \text {analog }\end{array}$ & $24 \mathrm{~h}$ & 19 & Kennedy et al. ${ }^{[17]}$ \\
\hline $\mathrm{NAD}^{+}$analog & $2 \mathrm{~d}$ & 26.8 & Zhou et al. ${ }^{[18]}$ \\
\hline 8-Br-NHD ${ }^{+}$ & $3 d$ & 59 & Wagner et al. ${ }^{[19]}$ \\
\hline 8-Chloro-NHD ${ }^{+}$ & Overnight & 46 & Potter et al. ${ }^{[10 \mathrm{c}]}$ \\
\hline 8-Iodo-NHD ${ }^{+}$ & Overnight & 62 & Potter et al. ${ }^{[10 \mathrm{c}]}$ \\
\hline 8-Ph-NAD ${ }^{+}$ & $48 \mathrm{~h}$ & 66 & Potter et al. ${ }^{[20]}$ \\
\hline 8-2'-deoxy-NAD ${ }^{+}$ & $48 \mathrm{~h}$ & 60 & Potter et al $^{[10]}$ \\
\hline $\mathrm{NAD}^{+}$analog & $17 \mathrm{~h}$ & 12.9 & Hou et al. ${ }^{[9]}$ \\
\hline
\end{tabular}

\section{$3.21 H$-四唑}

$1 H$-四唑在焦磷酸键的合成中具有广泛应用. $1 H$-四 唑是一种布朗斯特酸(Brønsted acid, $\left.\mathrm{p} K_{\mathrm{a}}=4.9\right)$, 具有一 定的酸性, 可以活化磷酰吗啉; 同时 $1 H$-四唑分子中含 有氮，可以作为亲核试剂进攻磷酰吗啉中间体形成活性 更高的中间体, 然后再与另外一分子磷酸单酯反应, 获 得焦磷酸产物.

Wong 等 ${ }^{[21]}$ 以 $1 H$-四唑为催化剂催化磷酰吗啉与磷 酸单酯的反应, 制备了 GDP-fucose, GDP-Mannose, UDP-Galactose (Scheme 12), 收率达 76\% 91\%. 并通 过 ${ }^{31} \mathrm{P}$ NMR 和质谱动态跟踪反应确定 $1 H$-四唑催化反应 机理(Scheme 13), $1 H$-四唑具有酸性和亲核性双重催化 特性，会生成更加活泼的中间体，加快反应进行.

Zhao 等 ${ }^{[22]}$ 以 $1 H$-四唑为催化剂合成了氟修饰糖的 糖焦磷酸核苷酯，产率在 $45 \%$ ～54\%之间(Scheme 14). 这些糖焦磷酸核苷酯被用以研究糖生物合成过程中酶 参与的反应机理.

Kosma 等 ${ }^{[23]}$ 发现从乙酰化糖磷酸单酯合成糖焦磷 酸核苷的产率要高于从羟基糖磷酸单酯. 从乙酰化糖磷 酸单酯偶联然后再脱乙酰基得到最终产物产率为 $90 \%$, 而差基糖磷酸单酯直接偶联产率仅为 $15 \%$ (Scheme 15). Williama 等 ${ }^{[24]}$ 也发现了类似的现象, 乙酰化糖磷酸单酯 为反应底物时, 最终产物的收率会有较大的提高. 可能 是乙酰化的糖磷酸单酯在吡啶中的溶解度要大于羟基 糖磷酸单酯, 利于反应的发生, 提高了反应收率.
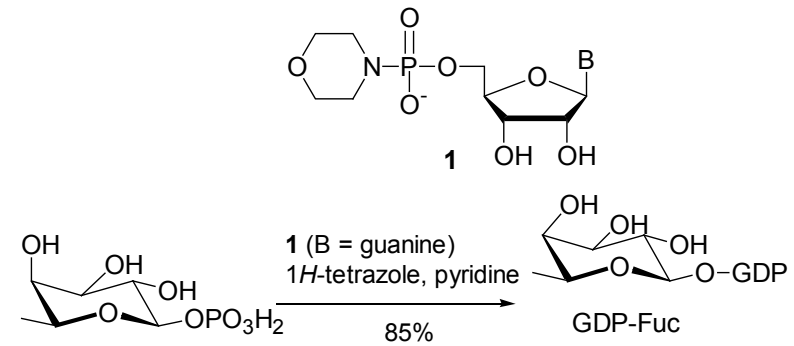

$\mathrm{Et}_{3} \mathrm{~N}$
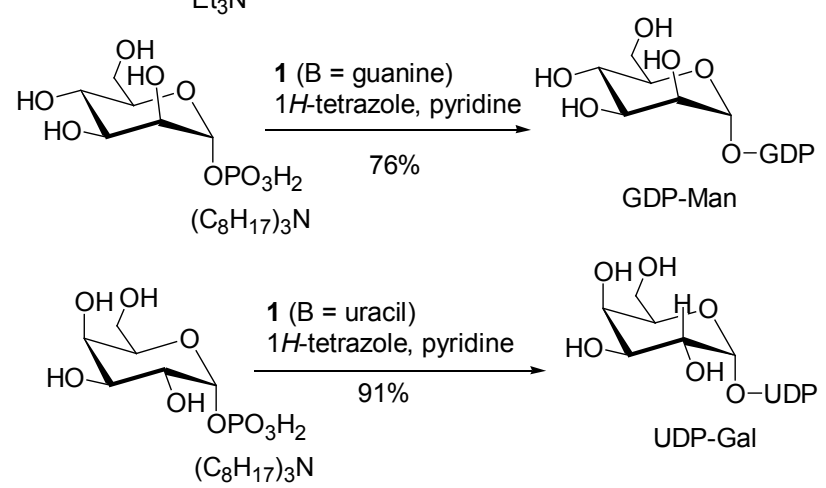

Scheme 12

我们课题组 ${ }^{[9]}$ 利用 $1 H$-四唑合成了 $\mathrm{NAD}^{+}$类似物, 产率 5.5\% (Eq. 3). 产率低的原因可能是烟酰胺单核苷 酸是一种内盐, 在吡啶中的溶解度不好, 导致产率低.

$1 H$-四唑催化反应具有高的反应活性，能够获得较 高产率的焦磷酸酯 ${ }^{[25]}$, 但是 $1 H$-四唑易爆炸，对操作条 件要求苛刻，限制了其应用.

\section{$4 \mathrm{PPh}_{3} /(\mathrm{PyS})_{2} / \mathrm{N}$-甲基咪唑}

$\mathrm{PPh}_{3} /(\mathrm{PyS})_{2}$ 具有很高的活性, $\mathrm{PPh}_{3} /(\mathrm{PyS})_{2}$ 氧化还原 离子对最早由 Mukaiyama 提出 ${ }^{[26]}$, 并用于活化羧酸、磷 酸以形成多肽及磷酸二酯，常用来合成多肽和 DNA， Abramova 等 ${ }^{[27]}$ 将其用于核苷酸三磷酸的合成, 取得了 较好的收率, 我们 ${ }^{[9,28]}$ 将这种氧化还原离子对应用于 $\mathrm{NAD}^{+}$类似物的合成(Scheme 16).

在此反应过程中, $\mathrm{PPh}_{3} /(\mathrm{PyS})_{2}$ 首先在 $N$-甲基咪唑存 在情况下活化磷酸单酯形成 $\mathrm{P}-\mathrm{N}^{+}$中间体, $\mathrm{P}-\mathrm{N}^{+}$中间 体具有很高的反应活性， $\mathrm{P}-\mathrm{N}^{+}$键容易断裂，可以与另 外一分子磷酸单酯快速反应生成焦磷酸酯. 使用此种方 法合成 $\mathrm{NAD}^{+}$类似物活性中间体无需纯化分离, 活化时

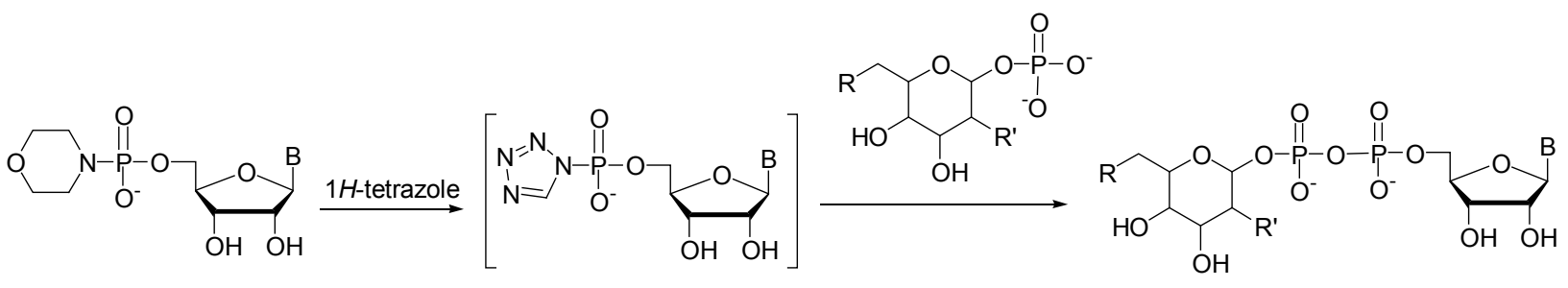

Scheme 13 

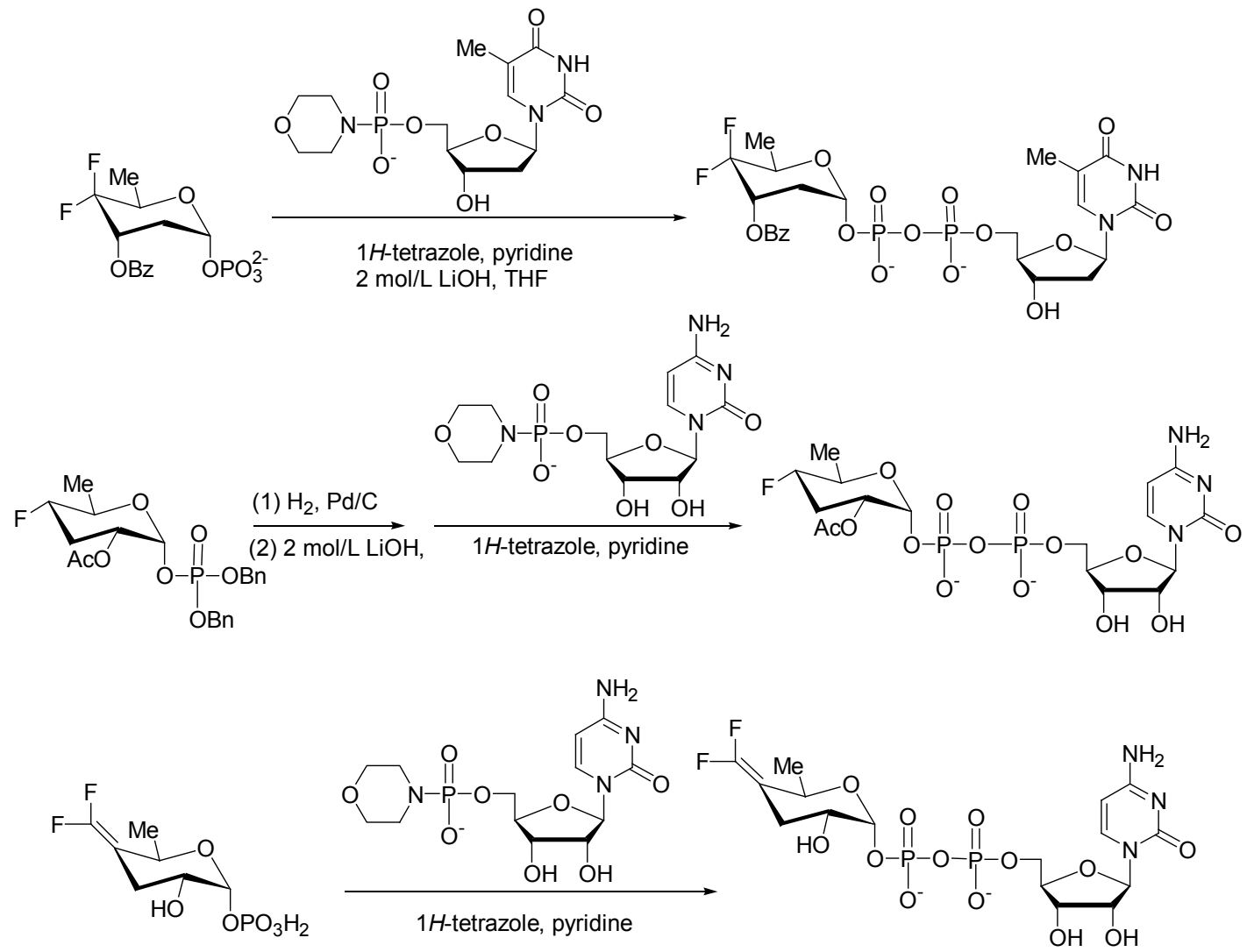

Scheme 14
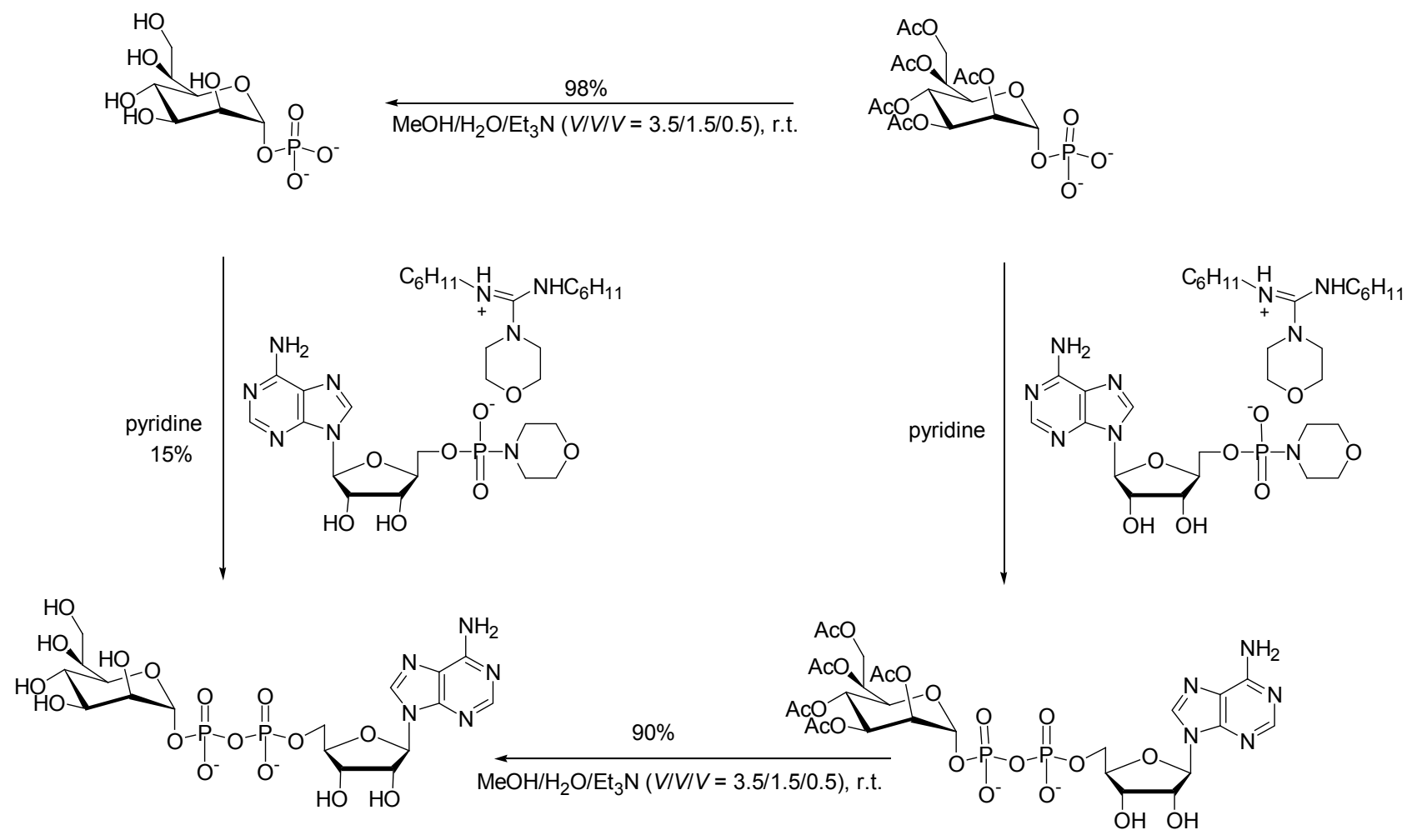

Scheme 15 


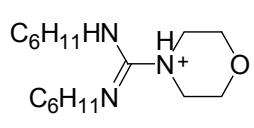<smiles>O=P([O-])(OCCc1ccccc1)N1CCOCC1</smiles>

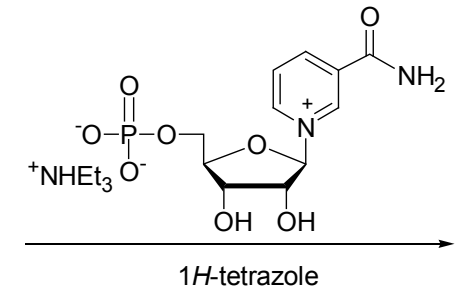<smiles></smiles>

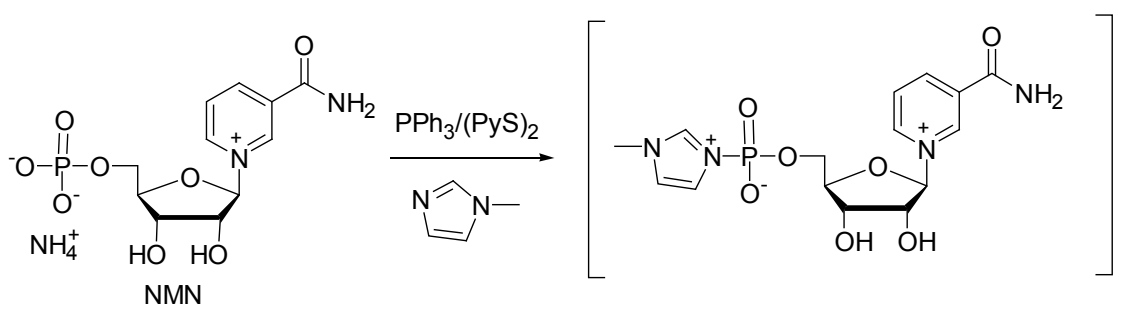
NMN

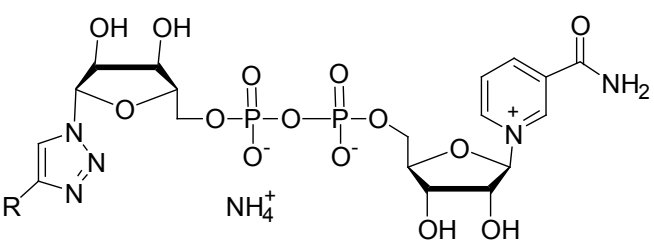

$\mathrm{R}=$<smiles>CCCCO</smiles>

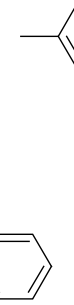<smiles>CCNC(C)=O</smiles><smiles>CCNC(=O)c1ccccc1</smiles><smiles>CC(=O)[O-]</smiles><smiles>CC(N)=O</smiles><smiles>Cc1ccccc1C</smiles>

Scheme 16

间 $15 \mathrm{~min}$, 反应时间 20,35 $\mathrm{min}$ 就可以完成反应, 可以 快速合成大量 $\mathrm{NAD}^{+}$类似物, 反应更加高效. 采用 $\mathrm{PPh}_{3} /(\mathrm{PyS})_{2}$ 活化, 在磷酸单酯存在条件下, 对羟基、氨 基等官能团无需经过保护和脱保护过程, 可以应用于糖 焦磷酸核苷酯的合成.

\section{DPCP 活化}

磷酰氯二苯酯具有较好的反应活性, 可以用来活化 磷酸单酯形成焦磷酸酯型活性中间体, 然后再与另外一 分子磷酸单酯反应获得焦磷酸酯产物 ${ }^{[29]}$. Potter 等 ${ }^{[30]}$ 利 用磷酰氯二苯酯活化 NMN, 然后与 AMP 类似物反应合 成 NAD 类似物(Scheme 17); Denu 等 ${ }^{[31]}$ 利用磷酰氯二苯 酯合成 $O$-乙酰化-ADP-核糖和 $N$-乙酰化类似物, 产率在 $5 \% \sim 20 \%$ 之间(Scheme 18).

\section{TFAA $/ N$-甲基咪唑}

Jakeman 等 ${ }^{[32]}$ 以三氟乙酸酐活化核酸, 然后与 $N$-甲 基咪唑反应生成 $\mathrm{P}-\mathrm{N}^{+}$活性中间体，与糖焦磷酸反应, $N-$ 甲基咪唑作为离去基团离去, 生成糖焦磷酸核苷酯. 合 成 UDP- $\alpha$ - $D$-glucose 等 7 种糖焦磷酸核苷酯, 产率在 $32 \% \sim 48 \%$ (Scheme 19).

此种方法采用三氟乙酸酩活化, 核糖中的羟基也参 与反应, 在后续反应中需要脱保护, 增加合成步骤.

\section{7 其它方法}

近年来发展了新的方法用以合成糖焦磷酸核苷酯, 以 $\mathrm{P}(\mathrm{III})$ 活化醇羟基, 利用 $\mathrm{P}(\mathrm{III})$ 高反应活性与另外一分 子单磷酸酯反应, 后经过氧化脱保护获得焦磷酸产物. 


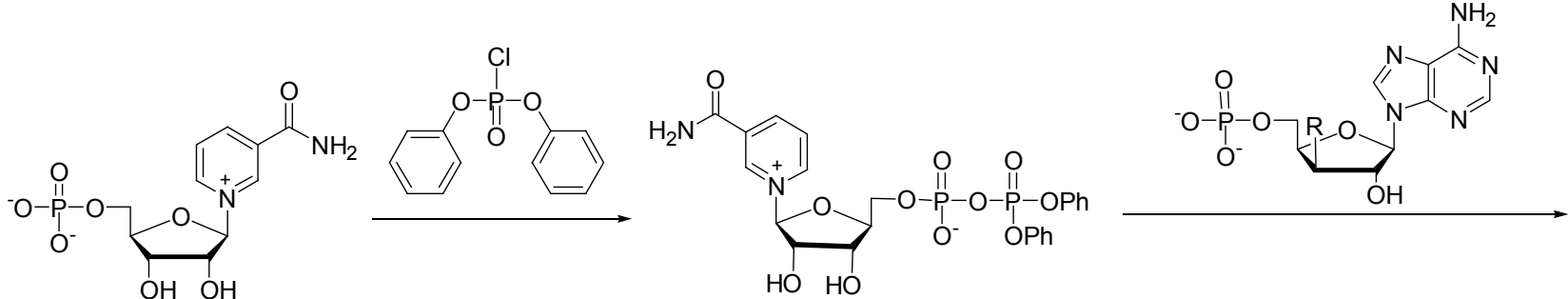

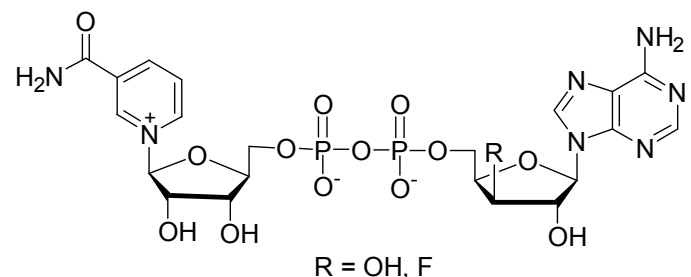

Scheme 17
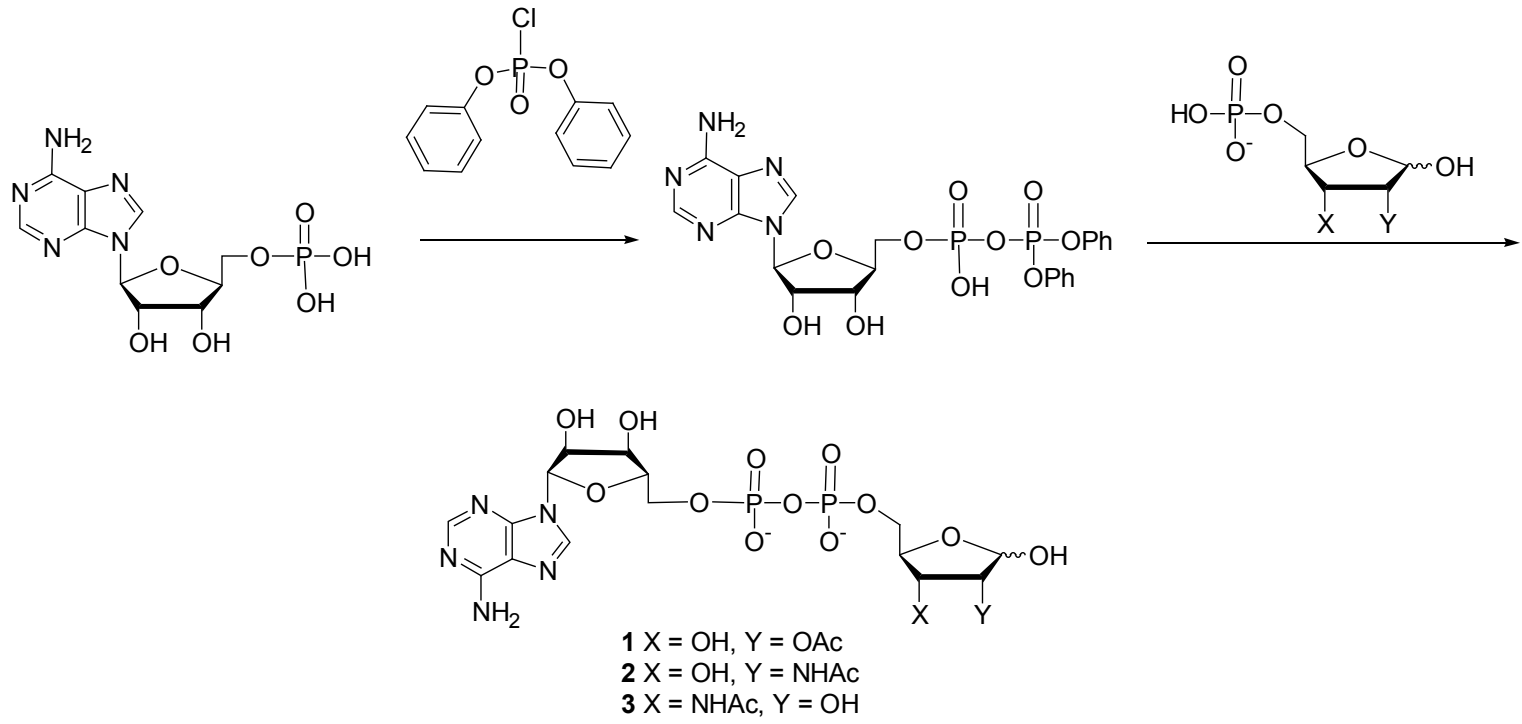

Scheme 18

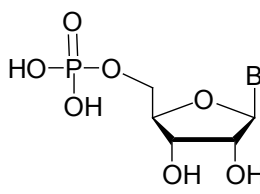

(1) TFAA, $\mathrm{Et}_{3} \mathrm{~N}, \mathrm{DMA}, \mathrm{MeCN}$

(2) $\mathrm{N}$-methylmidazole, $\mathrm{Et}_{3} \mathrm{~N}, \mathrm{MeCN}$

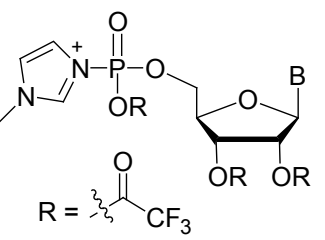

(1)

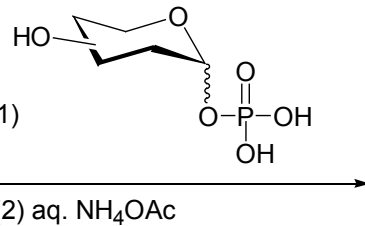

(2) aq. $\mathrm{NH}_{4} \mathrm{OAc}$

UDP- $\alpha-D$-glucose ADP- $\alpha$-L-rhamnose UDP- $\alpha$ - L-rhamnose ADP- $\beta$ - L-fucose UDP- $\beta$ - L-fucose ADP- $\alpha$ - L-arabinose UDP- $\alpha$-L-arabinose<smiles></smiles>

Scheme 19 


\section{1 亚磷酰胺}

Gold 等 ${ }^{[33]}$ 采用 2-㲵乙基- $N, N$-二异丙基氯代亚磷酰 胺与 2,3-二乙酰基尿苷反应获得 $2^{\prime}, 3^{\prime}$-二乙酰基尿苷$5^{\prime}$-(2-氧基乙基- $N, N$-二异丙基)亚磷酰胺, 然后与硅保护 的糖磷酸单酯反应, 磷酸-亚磷酸中间体经过 $t-\mathrm{BuOOH}$ 氧化, 经过一系列脱保护过程获得尿苷糖焦磷酸酯, 产 率在 $63 \% \sim 76 \%$ 之间(Eq. 4).

此种方法能够获得较高的收率, 但由于亚磷酰胺的 高反应活性, 需要对活化核苷中核糖 2,3 位羟基进行保 护，同时活性中间体尿苷亚磷酸胺可以与羟基进行反 应，所以糖磷酸单酯中的羟基在反应之前需要进行保 护，后经过脱保护完成产物的合成. 此种方法合成步骤 长, 涉及多步保护及脱保护过程, 且原料 2-氰乙基- $N, N$ 二异丙基氯代亚磷酰胺价格较高, 限制其应用.

\subsection{6 -硝基-2-氯-1,3,2-苯并二氧磷杂环己烷(5-nitro- cycloSal phosphochloridite)}

Meier 课题组 ${ }^{[34]}$ 首先采用三氯化磷合成 5-nitrocycloSal phosphochloridite, 然后与一分子的 2'-脱氧-3'-
乙酰基一胸苷反应, 氧化生成 5-nitro-cycloSal deoxythymidine 后, 最后与糖基-1-磷酸盐反应经脱保护得 到最终产物，产率 46\% 60\% (Scheme 20). 此种方法也 需要对反应分子中的羟基经过一系列保护和脱保护过 程.

\section{8 结论与展望}

近年来，随着人们对生命活动的探索，焦磷酸酯化 合物的合成研究迅速，其合成方法正不断得到改进和创 新，但其合成仍然存在很多问题及困难，如糖苷键容易 断裂; 磷酸根的存在降低了原料在有机溶剂中的溶解 度，而偶联反应一般需要无水条件，加大了反应的难度; 活化过程中易生成对称型焦磷酸酯产物; 糖中含有羟基 等活性官能团易于发生一系列副反应，涉及到官能团的 选择性保护和脱保护，增加反应步骤。而寻求温和反应 条件、探索降低副产物的合成方法及设计快速合成多样 化的产物结构将是未来主要关注方向.
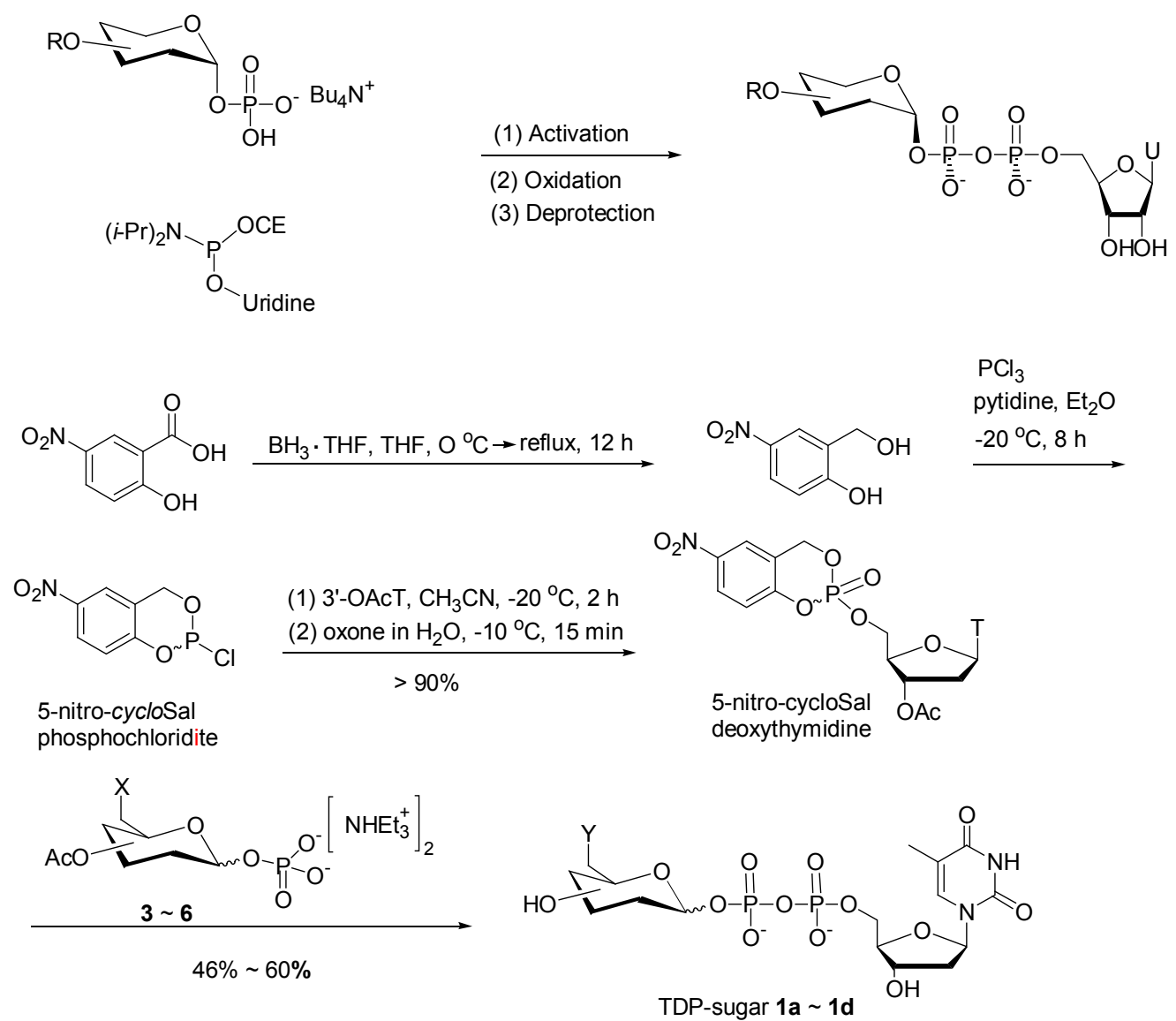

$$
\begin{aligned}
& 3 \sim 5 \mathrm{X}=\mathrm{OAc} ; 6 \mathrm{X}=\mathrm{H} \\
& 1 \mathrm{a} \sim 1 \mathrm{c} Y=\mathrm{OH} ; 1 \mathrm{~d} \mathrm{Y}=\mathrm{H}
\end{aligned}
$$

Scheme 20 


\section{References}

[1] (a) Lin, S. J.; Guarente, L. Curr. Opin. Cell Biol. 2003, 15, 241.

(b) Pollak, N.; Dolle, C.; Ziegler, M. Biochem. J. 2007, 402, 205.

(c) van der Donk, W. A.; Zhao, H. Curr. Opin. Biotechnol. 2003, 14, 421 .

(d) Sauve, A. A.; Deng, H. T.; Angeletti, R. H.; Schramm, V. L. J. Am. Chem. Soc. 2000, 122, 7855.

(e) Xia, W. L.; Wang, Z.; Wang, Q.; Han, J.; Zhao, C. P.; Hong, Y. Y.; Zeng, L. L.; Tang, L.; Ying, W. H. Curr. Pharm. Des. 2009, 15, 12.

[2] (a) Leloir, L. F. Science 1971, 172, 1299

(b) Kochetkov, N. K.; Shibaev, V. N. Adv. Carbohydr. Chem. Biochem. 1973, 28, 307.

[3] Mohnen, D. Curr. Opin. Plant Biol. 2008, 11, 266.

[4] Freeze, H. In Essentials of Glycobiology, Eds.: Varki, A.; Cummings, R.; Esko, J.; Freeze, H.; Hart, G.; Marth, J., Cold Spring Harbor Laboratory Press, New York, 1999, p. 66.

[5] (a) Thibodeaux, C. J.; Melançon, C. E.; Liu, H. W. Angew. Chem., Int. Ed. 2008, 47, 9814.

(b) Bourgeaux, V.; Piller, F.; Piller, V. Bioorg. Med. Chem. Lett. 2005, 15,5459 .

[6] Wagner, G. K.; Pesnot, T.; Field, R. A. Nat. Prod. Rep. 2009, 26, 1172 .

[7] Hughes, N. H.; Kenner, G. W.; Todd, A. C. J. Chem. Soc. 1957, 3733.

[8] Dowden, J.; Moreau, C.; Brown, R. S.; Berridge, G.; Galione, A.; Potter, B. V. L. Angew. Chem., Int. Ed. 2004, 43, 4637.

[9] Hou, S. H.; Liu, W. J.; Zhao, Z. B. Chin. J. Org. Chem. 2012, 32, 349 (in Chinese).

(侯淑华, 刘武军, 赵宗保, 有机化学 2012, 32, 349.)

[10] (a) Koh, D. W.; Coyle, D. L.; Mehta, N.; Ramsinghani, S.; Kim, H.; Slama, J. T.; Jacobson, M. K. J. Med. Chem. 2003, 46, 4322.

(b) Franchetti, P.; Cappellacci, L.; Pasqualini, M.; Petrelli, R.; Jayaprakasan, V.; Jayaram, H. N.; Boyd, D. B.; Jain, M. D.; Grifantini, M. Bioorg. Med. Chem. 2005, 13, 2045.

(c) Moreau, C.; Wagner, G. K.; Weber, K.; Guse, A. H.; Potter, B. V. J. Med. Chem. 2006, 49, 5162.

(d) Das, A.; Ko, H.; Burianek, L; Barrett, M.; Harden, T.; Jacobson, K. J. Med. Chem. 2010, 53, 471.

[11] (a) Kurosu, M.; Mahapatra, S.; Narayanasamy, P.; Crick, D. C. Tetrahedron Lett. 2007, 48, 799.

(b) Takaku, H.; Sato, J.; Ishida, H.-K.; Inazu, T.; Ishida, H.; Kiso, M. Glycoconjugate J. 2006, 23, 565.

(c) Ishimizu, T.; Uchida, T.; Sano, K.; Hase, S. Tetrahedron: Asymmetry 2005, 16, 309.

(d) Pesnot, T.; Wagner, G. K. Org. Biomol. Chem. 2008, 6, 2884.

(e) Ko, H.; Fricks, I.; Ivanov, A. A.; Harden, T. K.; Jacobson, K. A. J. Med. Chem. 2007, 50, 2030.

[12] Wahler, D.; Reymond, J.-L. Can. J. Chem. 2002, 80, 665.

[13] Moffatt, J. G.; Khorana, H. G. J. Am. Chem. Soc. 1958, 80, 3756.

[14] (a) Roseman, S.; Distler, J. J.; Moffatt, J. G.; Khorana, H. G. J. Am. Chem. Soc. 1961, 83, 659.

(b) Moffatt, J. G.; Khorana, H. G. J. Am. Chem. Soc. 1961, 83, 649.

[15] Collier, A.; Wagner, G. K. Chem. Commum. 2008, 178.

[16] Lee, J.; Churchil, H.; Choi, W. B.; Lynch, J. E.; Roberts, F. E.; Volante, R. P.; Reider, P. J. Chem. Commun. 1999, 729.
[17] Kennedy, K. J.; Bressi, J. C.; Gelb, M. H. Bioorg. Med. Chem. Lett. 2001, 11, 95 .

[18] Zhou, G.-C.; Parikh, S. L.; Tyler, P. C.; Evans, G. B.; Furneaux, R. H.; Zubkova, O. V.; Benjes, P. A.; Schramm, V. L. J. Am. Chem. Soc. 2004, 126, 5690.

[19] Wagner, G. K.; Guse, A. H.; Potter, B. V. J. Org. Chem. 2005, 70, 4810 .

[20] Zhang, B.; Wagner, G.; Weber, K.; Garnham, C.; Morgan, A.; Galione, A.; Guse, A.; Potter, B. J. Med. Chem. 2008, 51, 1623.

[21] Wittmann, V.; Wong, C. H. J. Org. Chem 1997, 62, 2144.

[22] (a) Zhao, Z.; Liu, H.-W. J. Org. Chem. 2001, 66, 6810.

(b) Chen, H.; Zhao, Z.; Hallis, T. M.; Guo, Z.; Liu, H. W. Angew. Chem., Int. Ed. 2001, 113, 627.

(c) Hallis, T. M.; Zhao, Z.; Liu, H.-W. J. Am. Chem. Soc. 2000, 122, 10493.

[23] Zamyatina, A.; Gronow, S.; Oertelt, C.; Puchberger, M.; Brade, H.; Kosma, P. Angew. Chem., Int. Ed. 2000, 39, 4150.

[24] (a) Dinev, Z.; Wardak, A. Z.; Brownlee, R. T.; Williams, S. J. Carbohydr. Res. 2006, 341, 1743.

(b) Fairweather, J. K.; Him, J. L. K.; Heux, L.; Driguez, H.; Bulone, V. Glycobiology 2004, 14, 775 .

[25] (a) Dulcey, A. E.; Qasba, P. K.; Lamb, J.; Griffiths, G. L. Tetrahedron 2011, 67, 2013.

(b) Ravalico, F.; Messina, I.; Berberian, M.; James, S.; Migaud, M.; Vyle, J. Org. Biomol. Chem. 2011, 9, 6496.

(c) Tsukamoto, H.; Kahne, D. Bioorg. Med. Chem. Lett. 2011, 21, 5050 .

(d) Wu, M.; Meng, Q.; Ge, M.; Bai, L.; Zhou, H. Tetrahedron Lett. 2011, 52, 5799.

(e) Tedaldi, L.; Pierce, M.; Wagner, G. Carbohydr. Res. 2012, 364, 22.

[26] Mukaiyama, T. Phosphorus Sulfur 1976, 1, 371

[27] Abramova, T. V.; Vasileva, S. V.; Serpokrylova, I. Y.; Kless, H.; Silnikov, V. N. Bioorg. Med. Chem. 2007, 15, 6549.

[28] (a)Hou, S.; Liu, W.; Ji, D.; Wang, Q.; Zhao, Z. K. Tetrahedron Lett. 2011, 52, 5855 .

(b) Ji, D.; Wang, L.; Hou, S.; Liu, W.; Wang, J.; Wang, Q.; Zhao, Z. J. Am. Chem. Soc. 2011, 133, 20857.

(c) Ji, D.; Wang, L.; Liu, W.; Hou, S.; Zhao, Z. Sci. China Chem. 2013, 56, 296.

[29] (a) Michelson, A. Biochim. Biophys. Acta 1964, 91, 1.

(b) Ma, Q.-F.; Reynolds, M. A.; Kenyon, G. L. Bioorg. Chem. 1989 , 17, 194.

[30] Mort, C. J. W.; Migaud, M. E.; Galione, A.; Potter, B. V. L. Bioorg. Med. Chem. 2004, 12, 475.

[31] Comstock, L. R.; Denu, J. M. Org. Biomol. Chem. 2007, 5, 3087.

[32] (a)Timmons, S. C.; Jakeman, D. L. Carbohydr. Res. 2008, 343, 865 .

(b) Mohamady, S.; Taylor, S. J. Org. Chem., 2011, 76, 6344.

[33] Gold, H.; van Delft, P.; Meeuwenoord, N.; Codée, J. D.; Filippov, D. V.; Eggink, G.; Overkleeft, H. S.; van der Marel, G. A. J. Org. Chem. 2008, 73, 9458.

[34] (a) Wolf, S.; Berrio, R. M.; Meier, C. Eur. J. Org. Chem. 2011, 2011, 6304.

(b) Wendicke, S.; Warnecke, S.; Meier, C. Angew. Chem., Int. Ed. 2008, 47, 1500 . 